\title{
Exploration of Indolo-imidazo[1,2-a]pyridine Compounds as Anti-Tubercular Agents through Docking, ADMET and Drug Likeliness Studies
}

\author{
Surabhi Jain $1, * \mathbb{C}$, Dhrubo Jyoti Sen ${ }^{2}(\mathbb{D})$ \\ 1 Faculty of Pharmacy, B. pharmacy College Rampura-kakanpur, (Gujarat Technological University), Panchmahals, \\ Gujarat, India; surabhi.jaiin@yahoo.in; (S.J.) \\ 2 School of Pharmacy, Techno India University, Sector-V, EM-4, Salt Lake Campus, Kolkata-700091, West Bengal, India; \\ dhrubosen69@yahoo.com (D.J.S.); \\ * Correspondence: surabhi.jaiin@yahoo.in; (S.J.);
}

Scopus Author ID: 57209870135

Received: 5.08.2021; Revised: 29.08.2021; Accepted: 30.08.2021; Published: 4.09.2021

\begin{abstract}
The resistance of Mycobacterium to anti-Mycobacterial drugs is a key stumbling block in its treatment. Exploration of diverse targets, the hunt for new chemical scaffolds, and different approaches to tuberculosis cure are all needed in this context. For growth and survival, Mycobacteria require oxidative phosphorylation. In Electron Transport System, the cytochrome B (QcrB) component is important for the bc1 complex's function, which is a clinical target for Q203 (Telacebec). This work includes the docking, ADMET, and Drug Likeliness profiles of indolo-imidazo[1,2-a]pyridine compounds. The structural resemblance of molecules to Q203 is the rationale behind the investigation. The chosen molecules follow the Lipinski rule of five. Out of 15 molecules, A12 and A13 can be investigated as potential therapeutic candidates after a thorough analysis of molecular docking, binding affinity, and ADMET profile. We suggest that these candidates are more likely to be used as antiMycobacterial agents or as beginning leads for creating novel and potent tubercular agents based on potential findings.
\end{abstract}

Keywords: QcrB; ATP synthase; ADMET; docking; tuberculosis; Lipinski rule.

(c) 2021 by the authors. This article is an open-access article distributed under the terms and conditions of the Creative Commons Attribution (CC BY) license (https://creativecommons.org/licenses/by/4.0/).

\section{Introduction}

Tuberculosis (TB) is one of the most deadly illnesses [1]. Tuberculosis in humans is caused by Mycobacterium Tuberculosis, also known as Koch Bacillus. M. bovis, M. africanum, M. canetti, and M. microti are the other causative agents in this M. tuberculosis complex [2]. The current WHO-recommended chemotherapy regimen for drug-susceptible tuberculosis consists of a two-month intensive phase consisting of four drugs (isoniazid, pyrazinamide, rifampicin, and ethambutol), followed by a four-month continuous phase consisting of two drugs (commonly isoniazid and rifampicin) [3, 4]. Multidrug-resistant tuberculosis (MDR-TB) and Extensive drug-resistant tuberculosis (XDR-TB), which have resistance to at least three of the six classes of second-line anti-tuberculosis drugs (aminoglycosides, polypeptides, fluoroquinolones, thioamides, cycloserine, and para-aminosalycilic acid), have given this disease a new face, making it much more dreadful than before [5]. To tackle MDR-TB and XDR-TB and the fast-evolving breakneck type of TB known as fully or extremely drugresistant TB (XDR-TB), new medications and novel approaches are in urgent need [6-8]. 
Mycobacteria's energy metabolism, specifically the oxidative phosphorylation route of cellular respiration, has recently emerged as a potential target pathway in drug discovery [9-11]. In both its latent and dormant forms, Mycobacteria use the oxidative phosphorylation pathway to make ATP. They have an electron transport mechanism that may convert menaquinone to menaquinol using various electron donors such as succinate, fumarate, and others. An electron is transferred from menaquinol to reduce the terminal acceptor oxygen in the next step, either via the cytochrome bc1 complex or the cytochrome bd menaquinol oxidase. Finally, an electrochemical gradient is created across the cytoplasmic membrane under aerobic circumstances, which is subsequently exploited by the F1F0-ATP synthase to make ATP [1214]. QcrB [15] is a highly promising and attractive therapeutic target for M. tuberculosis because of its involvement in respiratory function. Telacebec or Q203 (6-chloro-2-ethyl-N-(4(4-(4- (trifluoromethoxy)phenyl)piperidin-1-yl) benzyl)imidazo [1,2-a]pyridine-3carboxamide) is an imidazo-pyridine derivative that inhibits the M. tuberculosis cytochrome bc1 complex, making it a first-in-class anti-tuberculosis drug [16-20]. Other substituted imidazopyridine derivatives from the literature [21-28] demonstrated imidazo-pyridine scaffold as effective anti-tubercular agents (Figure 1). The Rieske iron-sulfur protein A subunit (QcrA), cytochrome B subunit (QcrB), and cytochrome C subunit (QcrC) are the three (3) basic subunits of the cytochrome bc1 complex [29, 30]. The cytochrome B subunit (QcrB) has been regarded as the most important component of the bc1 complex because of its coordination with other components. In the present work, we analyzed drug likeliness, ADMET, and binding affinity through docking studies of various analogs of indolo-imidazo[1,2-a]pyridine as antitubercular agents.

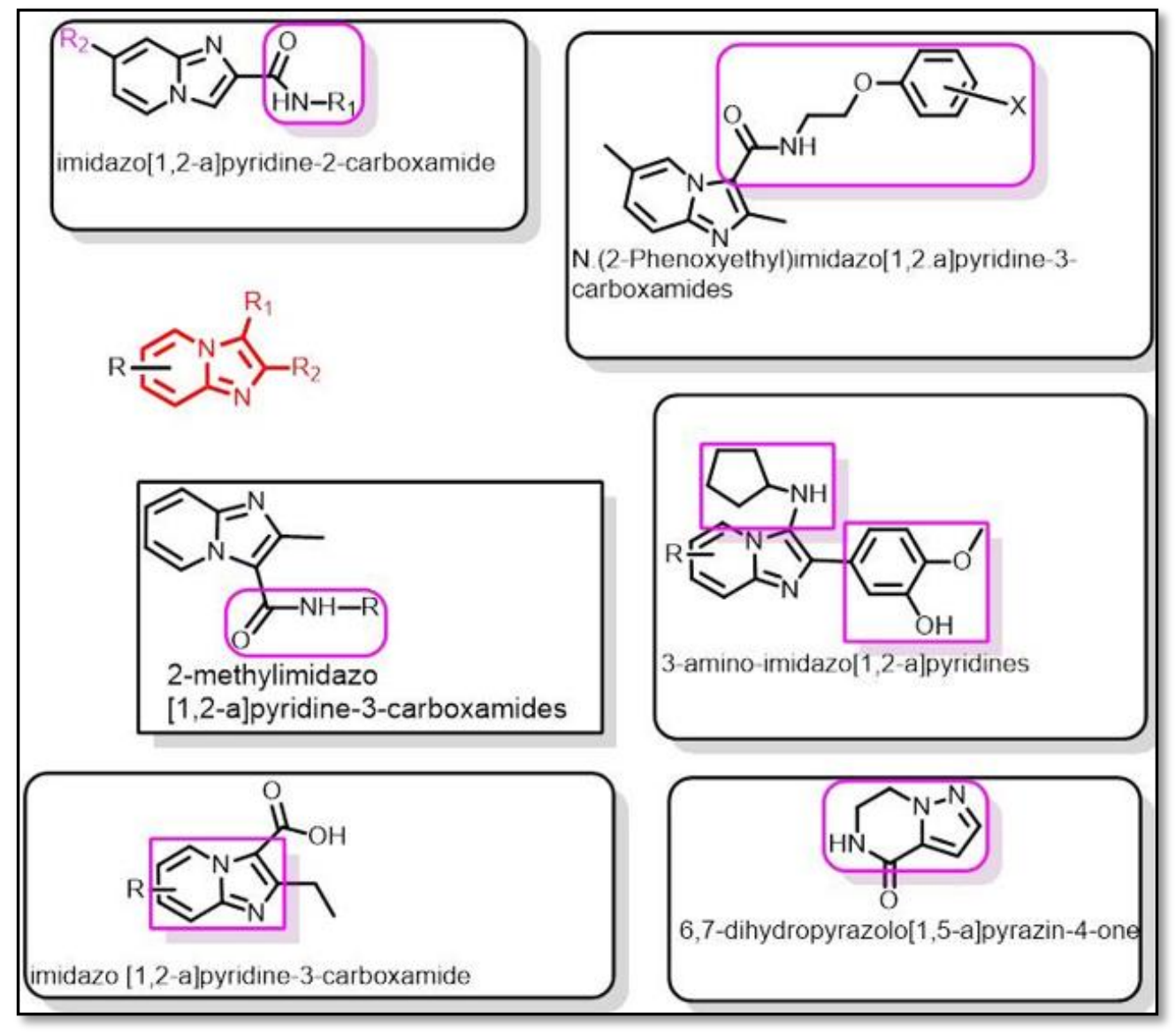

Figure 1. Different Imidazopyridine derivatives as Anti-tubercular agents. 


\section{Materials and Methods}

\subsection{Dataset.}

From the literature, 15 indolo-imidazo[1,2-a]pyridine derivatives were selected [31]. VEGAZZ version 3.2.1 was used for sketching and then minimization of molecules [32]. The software Autodock 4.0 was used to perform docking studies on these energy-minimized molecules [33].

\subsection{Protein preparation and molecular docking.}

The cytochrome B subunit is the most important part of the bc1 complex (QcrB). Because the 3D structure of QcrB was not present in the Protein Data Bank, an in-house homology modeled protein of QcrB was chosen as the protein [34]. Interacting residues from the literature $[35,36]$ were utilized as reference points to create a grid box around the active site. Using AutoDock's auto grid 4.0, grid maps of interaction energies with various atom types included in the ligands (A, C, HD, N, NA, OA, and SA) were generated. To cover all amino acid residues in the active pocket with $0.375 \AA$ spacing between grid points, the grid center was set to $-0.36,16.06$, and -18.90 for $\mathrm{x}, \mathrm{y}$, and $\mathrm{z}$ dimensions, respectively, and the grid box size was fixed to 60, 60, and 60 for $\mathrm{x}, \mathrm{y}$, and $\mathrm{z}$ dimensions, respectively (Supplementary Figure S1). With a maximum of 2,50,000 assessments, the Lamarckian Genetic Algorithm was chosen as the searching parameter. Autodock tools 1.5.6 and Discovery Studio visualizer was used to evaluate and visualize the docking findings [37, 38].

\subsection{Lipinski's rule and ADMET prediction.}

To estimate the physicochemical properties and several pharmacokinetic parameters of the selected compounds in this research, the Swiss ADME [39] prediction (http://www.swissadme.ch/) and PreADME-T server (https://preadmet.bmdrc.kr/adme/) [40] were employed. The Swiss ADME prediction was used to calculate the molecular weight (Mol. Wt.), number of rotatable bonds (NRB), number of hydrogen bond acceptors (HA), number of hydrogen bond donors(HD), Topological polar surface area (TPSA), and Log Po/w (iLOGP), and the PreADME-T server was used to assess human intestinal absorption (HIA), plasma protein binding, carcinogenicity, and inhibitory capacity of selected compounds (CYP 2D6, CYP 2C19, and CYP 3A4 subtypes).

\section{Results and Discussion}

\subsection{Ligand preparation.}

The selected indolo-imidazo[1,2-a]pyridine derivatives and Q203 (Figure 2.) was converted in AutoDock useful format .pdbqt after the addition of partial charges ' $q$ ' and atom types ' $t$ '. They were kept flexible, with all rotatable bonds active.

\subsection{Molecular docking.}

A three-dimensional grid was built around interacting residues with a distance of $5 \AA$. Leu174, Pro306, Ser304, Leu180, Glu314, and Thr313 are some of the residues that cover the active site [35, 36]. Q203's NH and Glu314 form a typical H-bond, as do other amino acid residues Ala179, 178, 302, Pro306, and Val338 that have $\pi$-alkyl interactions. Ser304, Thr313, 
Ala 317, and Met310 all show Van der Waal interactions. The fluorine in Q203 interacts with Phe199, Asp202, Gly200, and 201. As a result, the created grid produced reliable results in line with the literature (Figure 3). As a result, the same dimensions were used for docking. Table 1 shows the results of the binding energy of all docked compounds and standard Inhibitor Q203. Compound no. A13 shown the highest docking score with $-7.36 \mathrm{Kcal} / \mathrm{mol}$ and the lowest score by A6 with $-6.19 \mathrm{Kcal} / \mathrm{mol}$ (Figure 4). A13 was accompanied by Glu314, which had a typical H-bond at a distance of $4.53 \AA$. Ser182 (3.06 ) exhibited yet another carbon-hydrogen bond. Thr313 interacts with an amide pi-stacked bond, Ala179 and Val338 engage with a pi-sigma bond, and Pro335, Ala317, and Ala318 connect with a pi-alkyl bond. With Glu314, the secondhighest scorer A6 clearly showed pi-anion interaction. Pro335, Ala317, and Ala318 interact with a pi-alkyl bond, whereas Thr313 interacts with an amide pi-stacked interaction. Supplementary Figure S2 summarises the binding interactions of other molecules (A111,A14,A15) with protein.

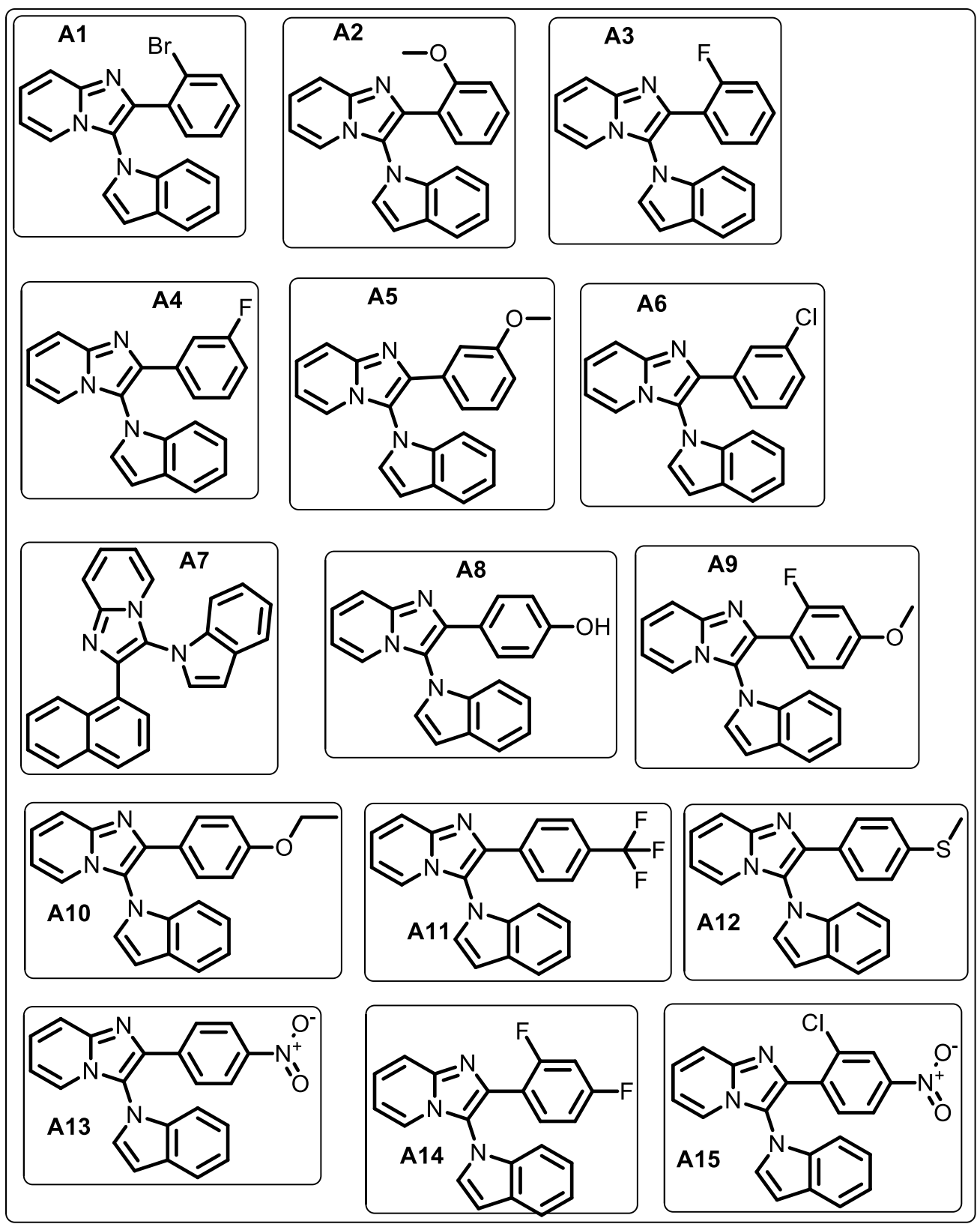

Figure 2. 2D structure of selected molecules (A1-A15). 


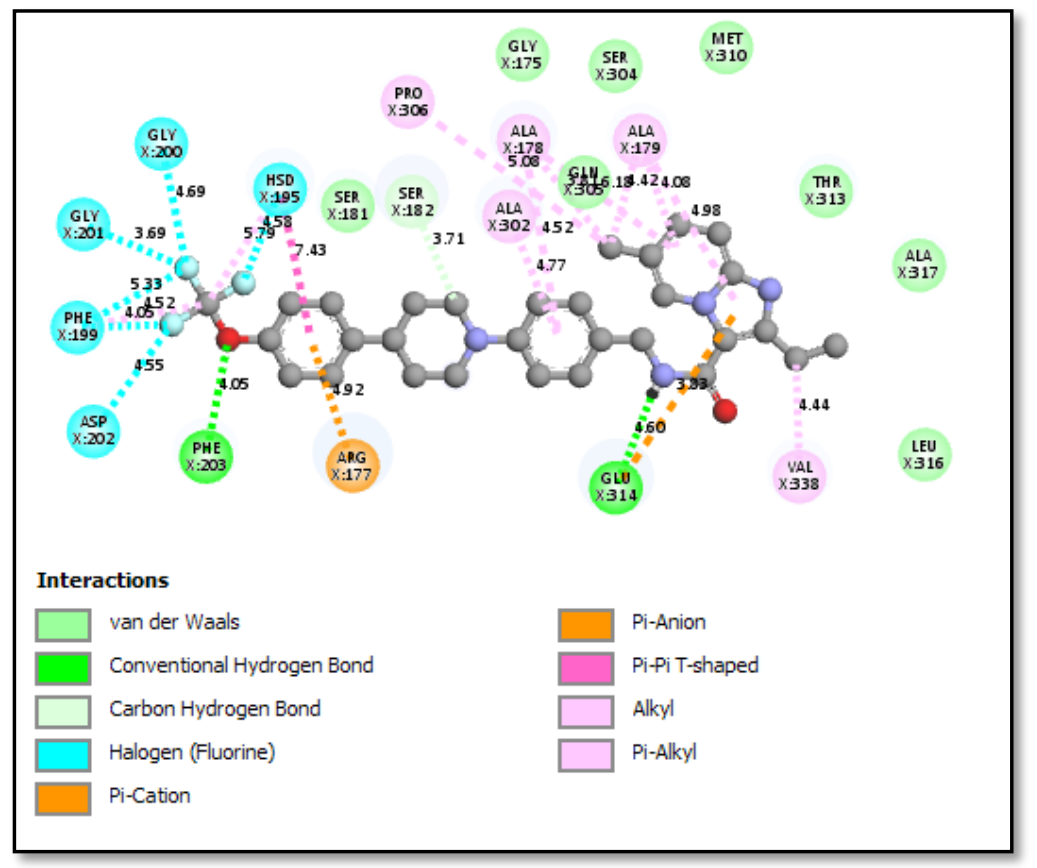

Figure 3. 2D interaction of Q203 molecule in active site pocket of QcrB protein.

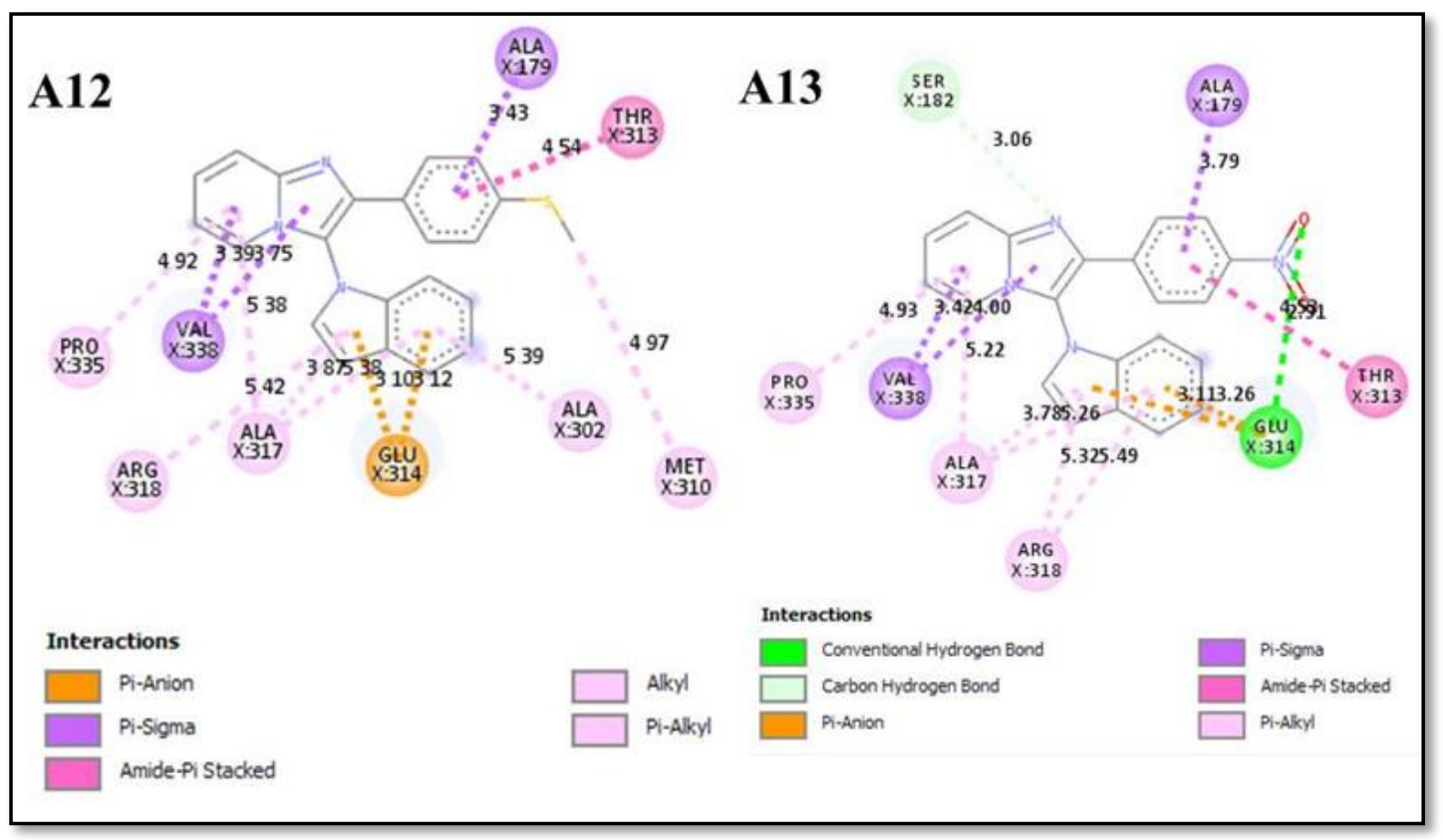

Figure 4. 2D interaction of best-docked compounds A12 and A13 in active site pocket of QcrB protein.

Table 1. Binding Energy of all compounds (A1 to A15) along with standard drug Q203.

\begin{tabular}{l|l|c} 
C. No. & IUPAC name & Docking Score (Kcal/mol) \\
\hline A1 & 2-(2-bromophenyl)-3-(1H-indol-1-yl)imidazo[1,2-a]pyridine & -6.64 \\
\hline A2 & 3-(1H-indol-1-yl)-2-(2-methoxyphenyl)imidazo[1,2-a]pyridine & -6.71 \\
\hline A3 & 2-(2-fluorophenyl)-3-(1H-indol-1-yl)imidazo[1,2-a]pyridine & -7.25 \\
\hline A4 & 2-(3-fluorophenyl)-3-(1H-indol-1-yl)imidazo[1,2-a]pyridine & -6.65 \\
\hline A5 & 3-(1H-indol-1-yl)-2-(3-methoxyphenyl)imidazo[1,2-a]pyridine & -6.66 \\
\hline A6 & 2-(3-chlorophenyl)-3-(1H-indol-1-yl)imidazo[1,2-a]pyridine & -6.19 \\
\hline A7 & 3-(1H-indol-1-yl)-2-(naphthalen-1-yl)imidazo[1,2-a]pyridine & -6.58 \\
\hline A8 & 4-(3-(1H-indol-1-yl)imidazo[1,2-a]pyridine-2-yl)phenol & -6.27 \\
\hline A9 & 2-(2-fluoro-4-methoxyphenyl)-3-(1H-indol-1-yl)imidazo[1,2-a]pyridine & -6.57 \\
\hline A10 & 2-(4-ethoxyphenyl)-3-(1H-indol-1-yl)imidazo[1,2-a]pyridine & -7.27 \\
\hline A11 & 3-(1H-indol-1-yl)-2-(4-(trifluoromethyl)phenyl)imidazo[1,2-a]pyridine & -6.69 \\
\hline A12 & 3-(1H-indol-1-yl)-2-(4-(methylthio)phenyl)imidazo[1,2-a]pyridine & -7.28 \\
\hline A13 & 3-(1H-indol-1-yl)-2-(4-nitrophenyl)imidazo[1,2-a]pyridine & -7.36 \\
\hline A14 & 2-(2,4-difluorophenyl)-3-(1H-indol-1-yl)imidazo[1,2-a]pyridine & -6.88
\end{tabular}




\begin{tabular}{l|l|c} 
C. No. & IUPAC name & Docking Score (Kcal/mol) \\
\hline A15 & 2-(2-chloro-4-nitrophenyl)-3-(1H-indol-1-yl)imidazo[1,2-a]pyridine & -6.80 \\
\hline Q203 & $\begin{array}{l}\text { 6-chloro-2-ethyl-N-(4-(4-(4-(trifluoromethoxy)phenyl)piperidin-1- } \\
\text { yl)benzyl)imidazo[1,2-a]pyridine-3-carboxamide }\end{array}$ & -7.70
\end{tabular}

\subsection{Lipinski's rule and ADMET prediction.}

The Swiss ADME prediction service (http://www.swissadme.ch/) was used to calculate Lipinski's Rule of Five drug-likeness features. Molecular weight must be less than 500 daltons, the number of $\mathrm{H}$-bond acceptors must be fewer than 10, the number of $\mathrm{H}$-bond donors must be less than 5, and a Log P must be less than five. Compounds' clinical efficacy depends on their ADMET profile (absorption, distribution, metabolism, elimination, and toxicity). Using the PreADMET server, users can forecast ADMET attributes using an easy-to-use web interface. Drug absorption is one of the ADMET variables that affect bioavailability. The compounds were discovered to be non-inhibitors for CYP2C19 and CYP3A4, as well as non-substrates for CYP2D6. For all substances, the projected Human intestinal absorption (HIA) was greater than 95\%. In terms of Plasma Protein Binding, all molecules are strongly bound (PPB). The carcinogenicity of mice and rats was predicted using a rodent carcinogenicity model based on data from the National Toxicology Program and the US Food and Drug Administration over two years. The compounds A15, A12, A11, A10, A9, A8, A7, A6, A5, and A2 do not cause cancer in rats. Compounds A10, A9, A8, and A5 have been shown to be non-carcinogenic in mice (Table 2).

Table 2. Molecular pharmacokinetic and ADME-T analysis of selected molecules from SwissADME and

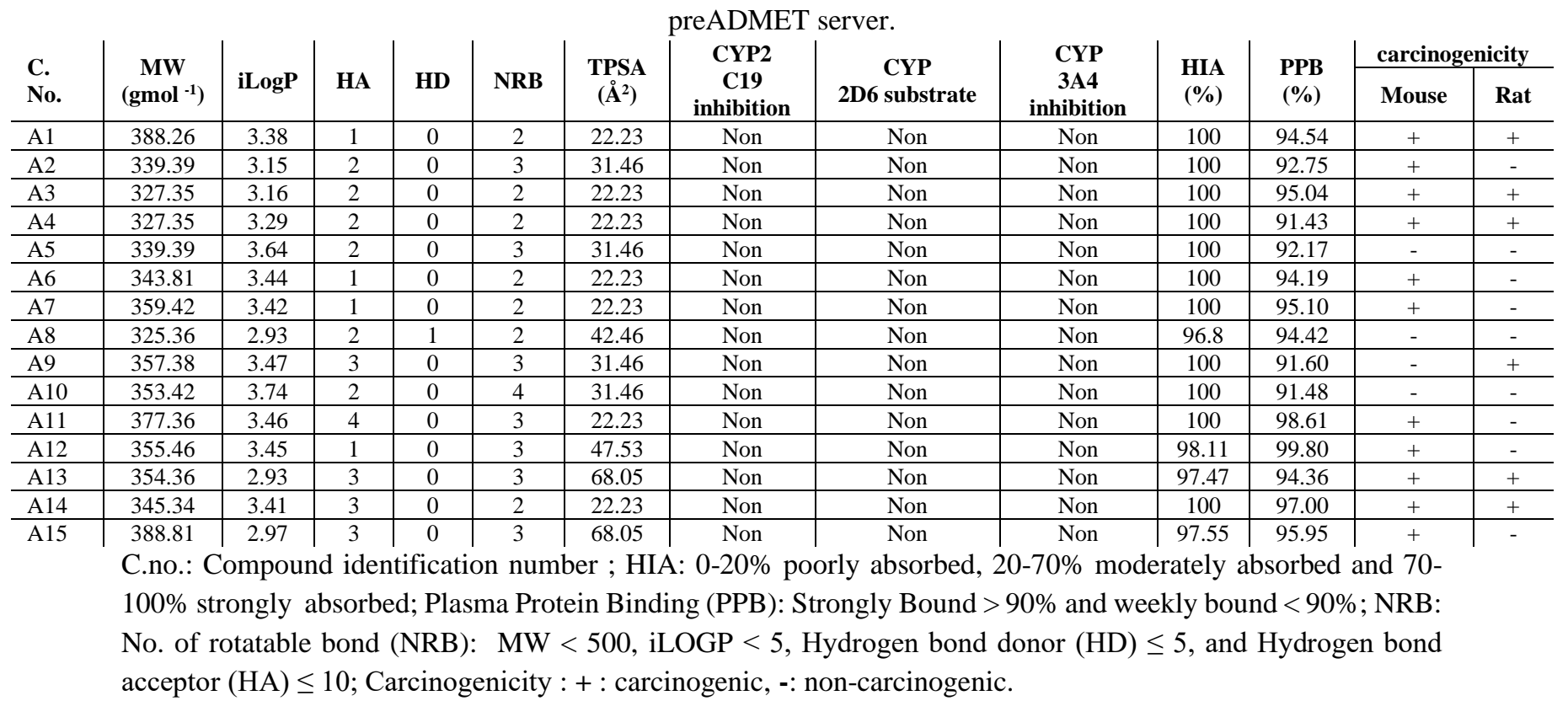

\section{Conclusions}

To sum up, we chose a dataset of 15 indolo-imidazo[1,2-a]pyridine derivatives, and they were docked to investigate their affinity for the QcrB protein. This cytochrome B (QcrB) subunit of the electron transport system is important for the function of the bc1 complex, and the therapeutic target is Q203 (Telacebec). The Lipinski rule of five holds for all molecules. We discovered that the docking scores of two compounds, A12 and A13, are remarkably similar to the conventional inhibitor Q203. They interacted with the same amino acid residues which are seen in standard inhibitors. The ADMET studies revealed that the majority of 
compounds are non-carcinogenic in rats and mice. We feel the selected compounds (A12 and A13) could be investigated further as possible lead molecules for rational drug design of QcrBinhibitors due to our evaluations. We expect that by combining medications that target the oxidative phosphorylation system components, we may develop an entirely new tuberculosis treatment regimen that will be successful against both drug-susceptible and multidrug-resistant tuberculosis.

\section{Funding}

This research received no external funding.

\section{Acknowledgments}

The first author (SJ) is thankful to B. Pharmacy College Rampura-Kakanpur, (Gujarat Technological University), Panchmahals, Gujarat, India.

\section{Conflicts of Interest}

The authors declare no conflict of interest.

\section{References}

1. Rawal, T.; Butani, S. Combating tuberculosis infection: A forbidding challenge. Indian J. Pharm. Sci. 2016, 78, https://doi.org/10.4103/0250-474x.180243.

2. Sirgel, F.A.; Tait, M.; Warren, R.M.; Streicher, E.M.; Böttger, E.C.; van Helden, P.D.; Gey van Pittius, N.C.; Coetzee, G.; Hoosain, E.Y.; Chabula-Nxiweni, M.; Hayes, C.; Victor, T.C.; Trollip, A. Mutations in the rrs A1401G Gene and Phenotypic Resistance to Amikacin and Capreomycin in Mycobacterium tuberculosis. Microbial Drug Resistance 2011, 18, 193-197, https://doi.org/10.1089/mdr.2011.0063.

3. van Heeswijk, R.P.G.; Dannemann, B.; Hoetelmans, R.M.W. Bedaquiline: a review of human pharmacokinetics and drug-drug interactions. Journal of Antimicrobial Chemotherapy 2014, 69, 2310-2318, https://doi.org/10.1093/jac/dku171.

4. Surabhi, J.; Smriti, S.; Dhrubo, J.S.; Saurabh, S.P. Enoyl-Acyl Carrier Protein Reductase (INHA): A Remarkable Target to Exterminate Tuberculosis. Anti-Infective Agents 2021, 19, 252-266, https://doi.org/10.2174/2211352518999201201114426.

5. Jain, A.; Mondal, R. Extensively drug-resistant tuberculosis: current challenges and threats. FEMS Immunology \& Medical Microbiology 2008, 53, 145-150, https://doi.org/10.1111/j.1574695X.2008.00400.x.

6. Velayati, A.A.; Farnia, P.; Masjedi. M.R. Letter to Editor: The totally drug resistant tuberculosis (TDR-TB ). Int. J. Clin. Exp. Med. 2013, 6, 307-9.

7. Lamont, E.A.; Dillon, N.A.; Baughn, A.D. The Bewildering Antitubercular Action of Pyrazinamide. Microbiol. Mol. Biol. Rev. 2020, 84, https://doi.org/10.1128/mmbr.00070-19.

8. Shetye, G.S.; Franzblau, S.G.; Cho, S. New tuberculosis drug targets, their inhibitors, and potential therapeutic impact. Translational Research 2020, 220, 68-97, https://doi.org/10.1016/j.trsl.2020.03.007.

9. Bald, D.; Villellas, C.; Lu, P.; Koul, A.; Rubin Eric, J.; Collier, R.J. Targeting Energy Metabolism in Mycobacterium tuberculosis, a New Paradigm in AntiMycobacterial Drug Discovery Dirk. MBio. 2017, 8, 1-11, https://doi.org/10.1128/mBio.00272-17.

10. Foo, C.S.; Pethe, K.; Lupien, A. Oxidative Phosphorylation-an Update on a New, Essential Target Space for Drug Discovery in Mycobacterium tuberculosis. Applied Sciences 2020, 10, https://doi.org/10.3390/app10072339.

11. Treatment of Highly Drug-Resistant Pulmonary Tuberculosis. New England Journal of Medicine 2020, 382, 2376-2377, https://doi.org/10.1056/nejmc2009939.

12. Cook Gregory, M.; Hards, K.; Dunn, E.; Heikal, A.; Nakatani, Y.; Greening, C.; Crick Dean, C.; Fontes Fabio, L.; Pethe, K.; Hasenoehrl, E.; Berney, M.; Jacobs Jr. William, R.; McShane, H.; Mizrahi, V.; Orme Ian, M. Oxidative Phosphorylation as a Target Space for Tuberculosis: Success, Caution, and Future Directions. Microbiology Spectrum 2017, 5, 1-22, https://doi.org/10.1128/microbiolspec.tbtb2-0014-2016.

13. Karan, G.; Mehul, P. Collocating Novel Targets for Tuberculosis (TB) Drug Discovery. Current Drug Discovery Technologies 2021, 18, 307-316, https://doi.org/10.2174/1570163817666200121143036. 
14. Beites, T.; O’Brien, K.; Tiwari, D.; Engelhart, C.A.; Walters, S.; Andrews, J.; Yang, H.-J.; Sutphen, M.L.; Weiner, D.M.; Dayao, E.K.; Zimmerman, M.; Prideaux, B.; Desai, P.V.; Masquelin, T.; Via, L.E.; Dartois, V.; Boshoff, H.I.; Barry, C.E.; Ehrt, S.; Schnappinger, D. Plasticity of the Mycobacterium tuberculosis respiratory chain and its impact on tuberculosis drug development. Nature Communications 2019, 10, https://doi.org/10.1038/s41467-019-12956-2.

15. Hasenoehrl, E.J.; Wiggins, T.J.; Berney, M. Bioenergetic Inhibitors: Antibiotic Efficacy and Mechanisms of Action in Mycobacterium tuberculosis. Frontiers in Cellular and Infection Microbiology 2021, 10, https://doi.org/10.3389/fcimb.2020.611683.

16. de Jager, V.R.; Dawson, R.; van Niekerk, C.; Hutchings, J.; Kim, J.; Vanker, N.; van der Merwe, L.; Choi, J.; Nam, K.; Diacon, A.H. Telacebec (Q203), a New Antituberculosis Agent. New England Journal of Medicine 2020, 382, 1280-1281, https://doi.org/10.1056/NEJMc1913327.

17. Lu, P.; Asseri, A.H.; Kremer, M.; Maaskant, J.; Ummels, R.; Lill, H.; Bald, D. The anti-mycobacterial activity of the cytochrome bcc inhibitor Q203 can be enhanced by small-molecule inhibition of cytochrome bd. Scientific Reports 2018, 8, https://doi.org/10.1038/s41598-018-20989-8.

18. ClinicalTrials.gov [Internet]. National Library of Medicine (US). 2019 September 10. Identifier NCT03563599, A Phase 2 Study to Evaluate Early Bactericidal Activity, Safety, Tolerability, and Pharmacokinetics of Multiple Oral Doses of Telacebec (Q203); [assessed 2021, May20] Available from: https://clinicaltrials.gov/ct2/show/NCT03563599.

19. Wang, J.; Jing, W.; Shi, J.; Huo, F.; Shang, Y.; Wang, F.; Chu, N.; Pang, Y. Bipolar Distribution of Minimum Inhibitory Concentration of Q203 Across Mycobacterial Species. Microbial Drug Resistance 2021, 27, 10131017, https://doi.org/10.1089/mdr.2020.0239.

20. Zhou, S.; Wang, W.; Zhou, X.; Zhang, Y.; Lai, Y.; Tang, Y.; Xu, J.; Yang, X.; Guddat, L.W.; Wang, Q.; Gao, Y.; Rao, Z.; Gong, H. Structure of mycobacterial cytochrome \&lt;em\&gt;bcc\&lt;/em\&gt; in complex with Q203 and TB47, two anti-TB drug candidates. bioRxiv 2021, https://doi.org/10.1101/2021.06.15.448498.

21. Jose, G.; Kumara, T.H.S.; Nagendrappa, G.; Sowmya, H.B.V.; Sriram, D.; Yogeeswari, P., et al. Synthesis, molecular docking and anti-mycobacterial evaluation of new imidazo[1,2-a]pyridine-2-carboxamide derivatives. Eur. J. Med. Chem. 2015, 89, 616-27. https://doi.org/10.1016/j.ejmech.2014.10.079.

22. Wu, Z.; Lu, Y.; Li, L.; Zhao, R.; Wang, B.; Lv, K.; Liu, M.; You, X. Identification of N-(2Phenoxyethyl)imidazo[1,2-a]pyridine-3-carboxamides as New Antituberculosis Agents. ACS Medicinal Chemistry Letters 2016, 7, 1130-1133, https://doi.org/10.1021/acsmedchemlett.6b00330.

23. Samala, G.; Nallangi, R.; Devi, P.B.; Saxena, S.; Yadav, R.; Sridevi, J.P.; Yogeeswari, P.; Sriram, D. Identification and development of 2-methylimidazo[1,2-a]pyridine-3-carboxamides as Mycobacterium tuberculosis pantothenate synthetase inhibitors. Bioorganic \& Medicinal Chemistry 2014, 22, 4223-4232, https://doi.org/10.1016/j.bmc.2014.05.038.

24. Odell, L.R.; Nilsson, M.T.; Gising, J.; Lagerlund, O.; Muthas, D.; Nordqvist, A.; Karlén, A.; Larhed, M. Functionalized 3-amino-imidazo[1,2-a]pyridines: A novel class of drug-like Mycobacterium tuberculosis glutamine synthetase inhibitors. Bioorganic \& Medicinal Chemistry Letters 2009, 19, 4790-4793, https://doi.org/10.1016/j.bmcl.2009.06.045.

25. Kang, S.; Kim, Y.M.; Kim, R.Y.; Seo, M.J.; No, Z.; Nam, K.; Kim, S.; Kim, J. Synthesis and structureactivity studies of side chain analogues of the anti-tubercular agent, Q203. European Journal of Medicinal Chemistry 2017, 125, 807-815, https://doi.org/10.1016/j.ejmech.2016.09.082.

26. Surase, Y.B.; Samby, K.; Amale, S.R.; Sood, R.; Purnapatre, K.P.; Pareek, P.K.; Das, B.; Nanda, K.; Kumar, S.; Verma, A.K. Identification and synthesis of novel inhibitors of mycobacterium ATP synthase. Bioorganic \& Medicinal Chemistry Letters 2017, 27, 3454-3459, https://doi.org/10.1016/j.bmcl.2017.05.081.

27. Pulipati, L.; Sridevi, J.P.; Yogeeswari, P.; Sriram, D.; Kantevari, S. Synthesis and anti-tubercular evaluation of novel dibenzo[b,d]thiophene tethered imidazo[1,2-a]pyridine-3-carboxamides. Bioorganic \& Medicinal Chemistry Letters 2016, 26, 3135-3140, https://doi.org/10.1016/j.bmcl.2016.04.088.

28. Montana, M.; Montero, V.; Khoumeri, O.; Vanelle, P. Quinoxaline Moiety: A Potential Scaffold against Mycobacterium tuberculosis. Molecules 2021, 26, https://doi.org/10.3390/molecules26164742.

29. Abdullahi, M.; Adeniji, S.E.; Arthur, D.E.; Haruna, A. Homology modeling and molecular docking simulation of some novel imidazo[1,2-a]pyridine-3-carboxamide (IPA) series as inhibitors of Mycobacterium tuberculosis. Journal of Genetic Engineering and Biotechnology 2021, 19, 1-13, https://doi.org/10.1186/s43141-020-00102-1.

30. Bahuguna, A.; Rawat, S.; Rawat, D.S. QcrB in Mycobacterium tuberculosis: The new drug target of antitubercular agents. Medicinal Research Reviews 2021, 41, 2565-2581, https://doi.org/10.1002/med.21779.

31. Ganesher, A.; Panda, G. TFA-catalysed tandem double cyclisation: A one-pot, metal-free routes for novel indolo-imidazo[1,2-a]pyridine derivatives. Tetrahedron Letters 2019, https://doi.org/10.1016/j.tetlet.2019.151317.

32. Pedretti, A.; Villa, L.; Vistoli, G. VEGA: a versatile program to convert, handle and visualize molecular structure on Windows-based PCs. Journal of molecular graphics \& modelling 2002, 21, 47-49, https://doi.org/10.1016/s1093-3263(02)00123-7. 
33. Morris, G.M.; Huey, R.; Lindstrom, W.; Sanner, M.F.; Belew, R.K.; Goodsell, D.S.; Olson, A.J. AutoDock4 and AutoDockTools4: Automated docking with selective receptor flexibility. Journal of computational chemistry 2009, 30, 2785-2791.

34. Jain, S.; Sharma, S.; Sen, D.J. Virtual screening, Docking, ADMET and Molecular Dynamics: A study to find Novel Inhibitors of Mycobacterium Tuberculosis targeting QcrB (under publication).

35. Thompson, A.M.; Denny, W.A. Chapter Four - Inhibitors of enzymes in the electron transport chain of Mycobacterium tuberculosis. In: Annual Reports in Medicinal Chemistry. Chibale, K. Ed.; Academic Press: Volume 52, 2019; pp. 97-130, https://doi.org/10.1016/bs.armc.2019.05.001.

36. Ko, Y.; Choi, I. Putative 3D Structure of QcrB from Mycobacterium tuberculosis Cytochrome bc1 Complex, a Novel Drug-Target for New Series of Antituberculosis Agent Q203. Bulletin of the Korean Chemical Society 2016, 37, 725-731, https://doi.org/10.1002/bkcs.10765.

37. Ionescu, M.I. Molecular docking investigation of the amantadine binding to the enzymes upregulated or downregulated in Parkinson's disease, ADMET \& DMPK 2020, 8, 149-175, https://doi.org/10.5599/admet.854.

38. BIOVIA D. S. BIOVIA Discovery Studio Visualizer, v16. 1.0. 15350, San Diego: Dassault Systemes; 2015.

39. Daina, A.; Michielin, O.; Zoete, V. SwissADME: A free web tool to evaluate pharmacokinetics, druglikeness and medicinal chemistry friendliness of small molecules. Sci. Rep. 2017, 7, 1-13, https://doi.org/10.1038/srep42717.

40. Lee, S.K.; Kang, Y.; Chang, G.S.; Lee, I.H.; Park, S.H.; Park, J. Bioinformatics and Molecular Design Research Center. Yonsei University, Seoul https://preadmet. bmdrc. kr 2017. 

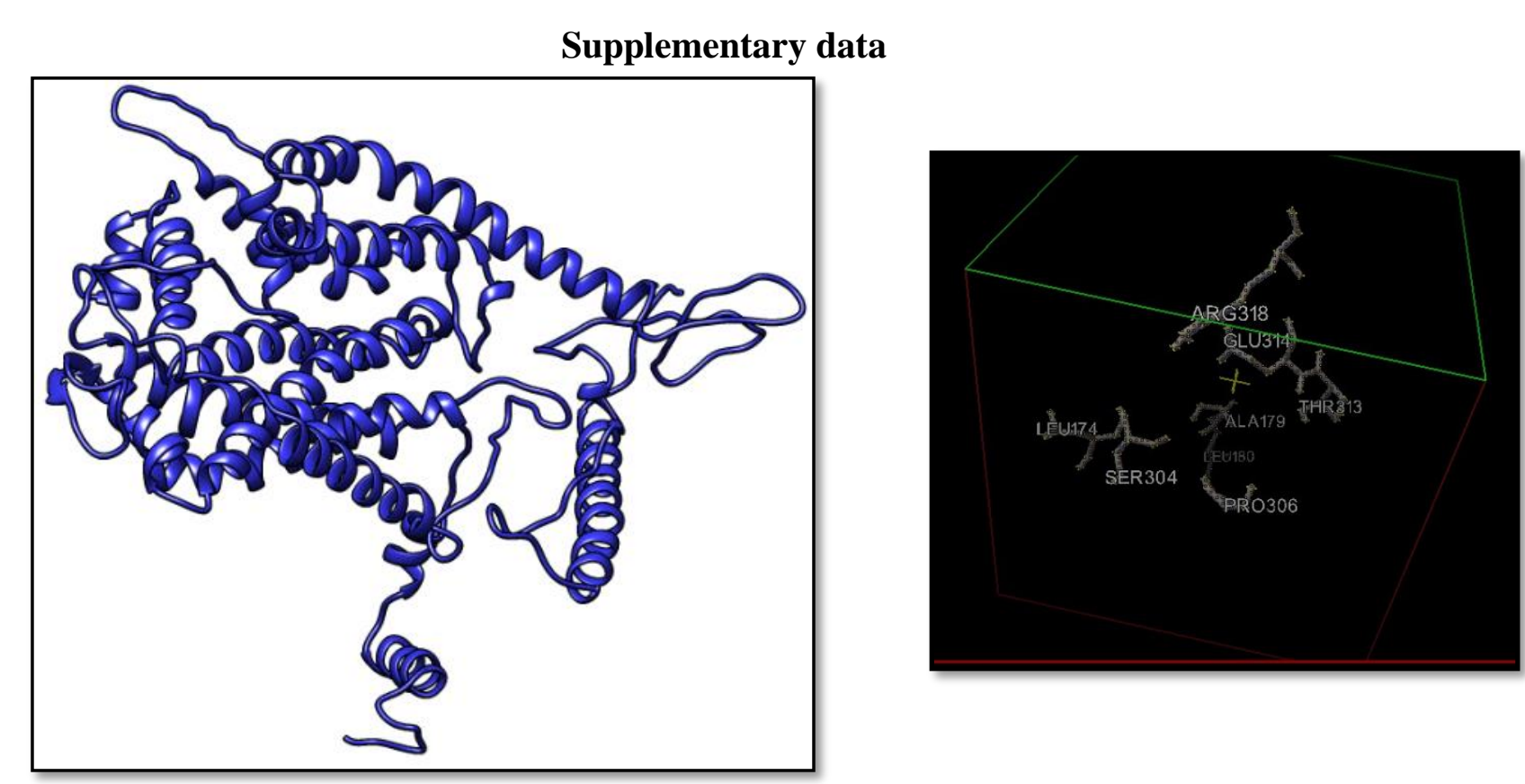

Figure S1. 3D structure of Protein (left) and Generated grid around important Amino Acid residues (right).

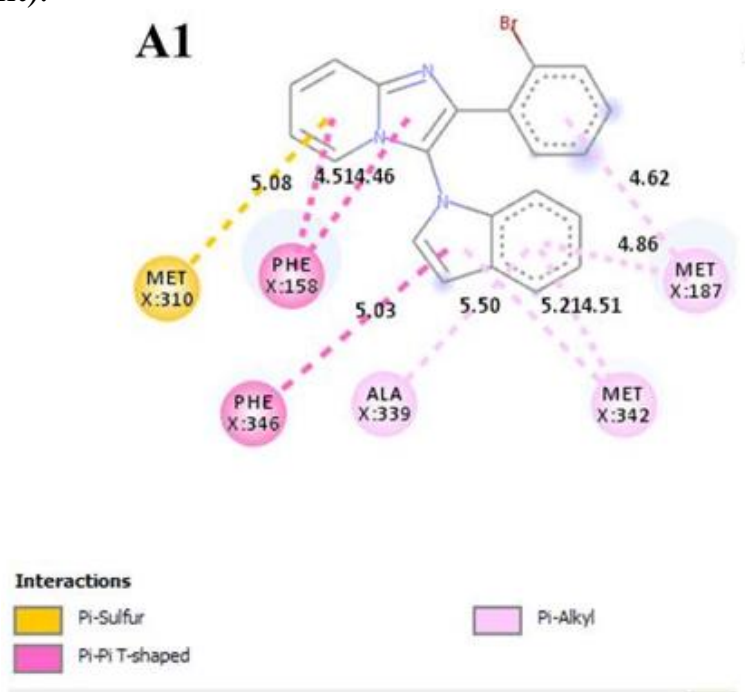

A2

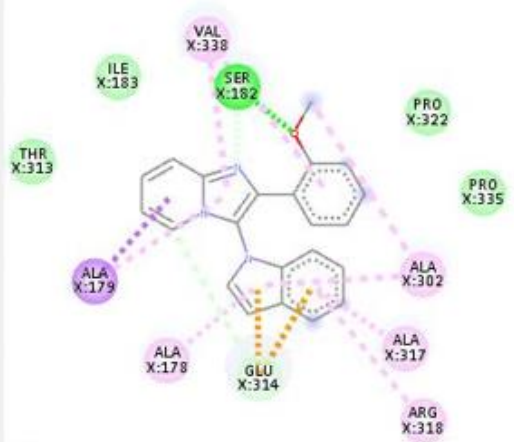

Interactions
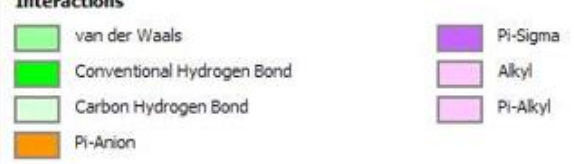

A4

A3
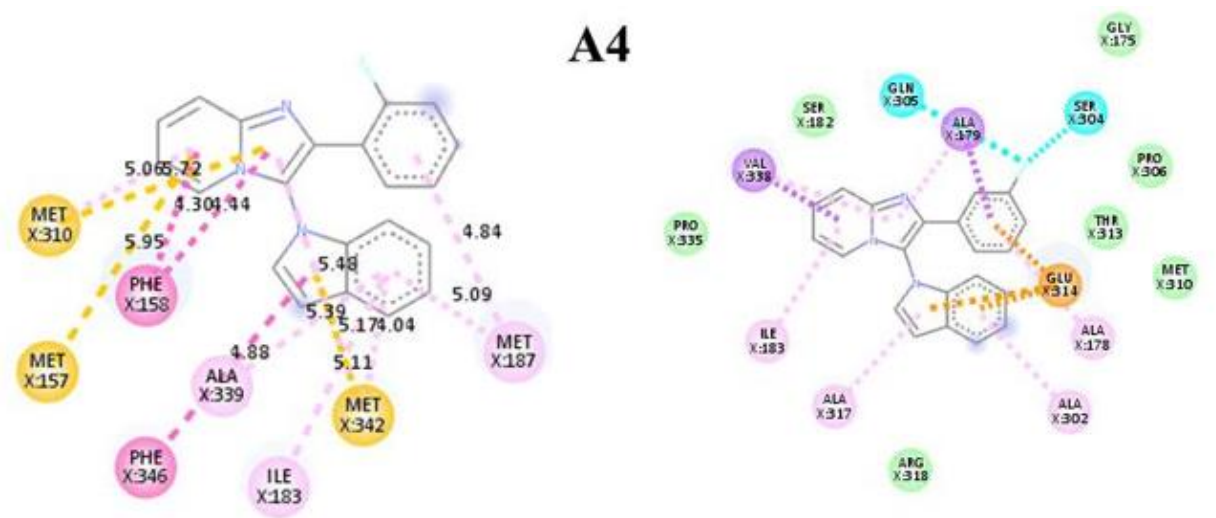

Interactions

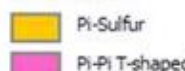

Pi-Alkyl

Interactions

$\square$ van der Waals

Halogen (Fluorine)

Pi-Anion 
A5

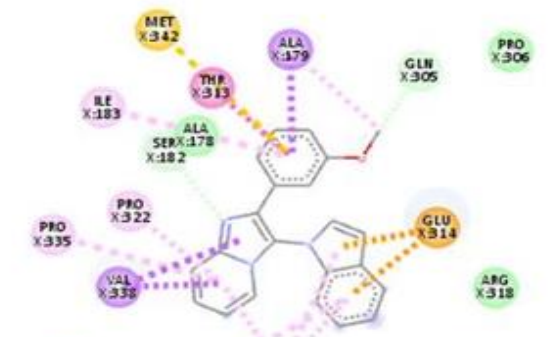

\section{A6}

xas $\times 334$

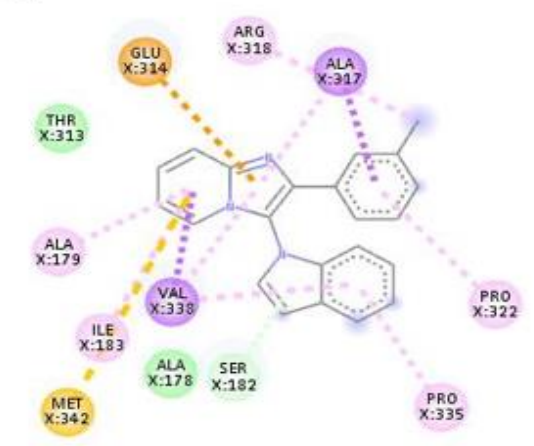

Interactions

$\square$ van der Waals
$\square$ Carbon Hydrogen Bond
$\square$ Pi-Anion
Pi-Sigma
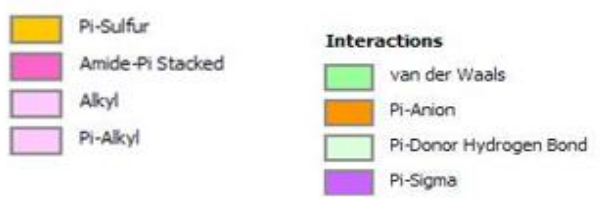

$\underset{\substack{\text { pRo } \\ x: 322}}{x}$

$\underset{335}{\substack{R 0 \\ x_{3}}}$

\section{A7}

\section{A8}
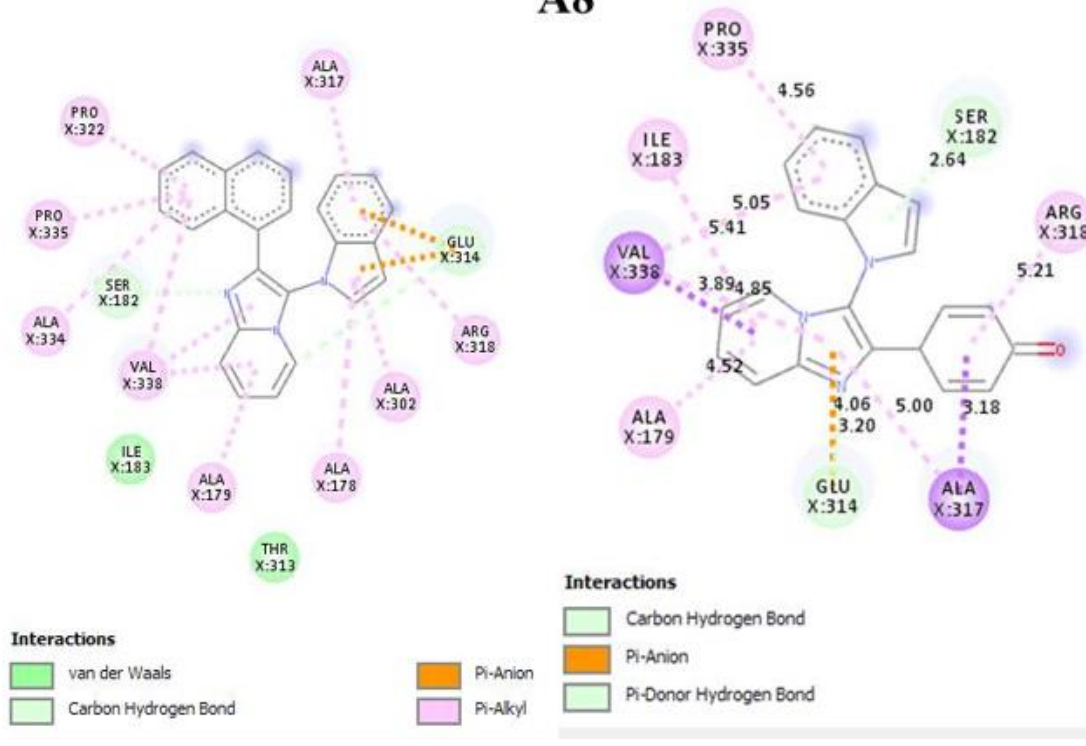

Interactions

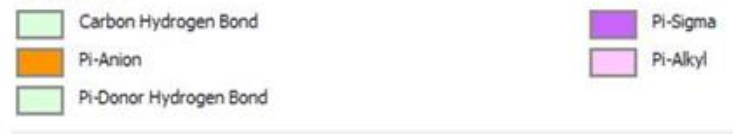

\section{A11}
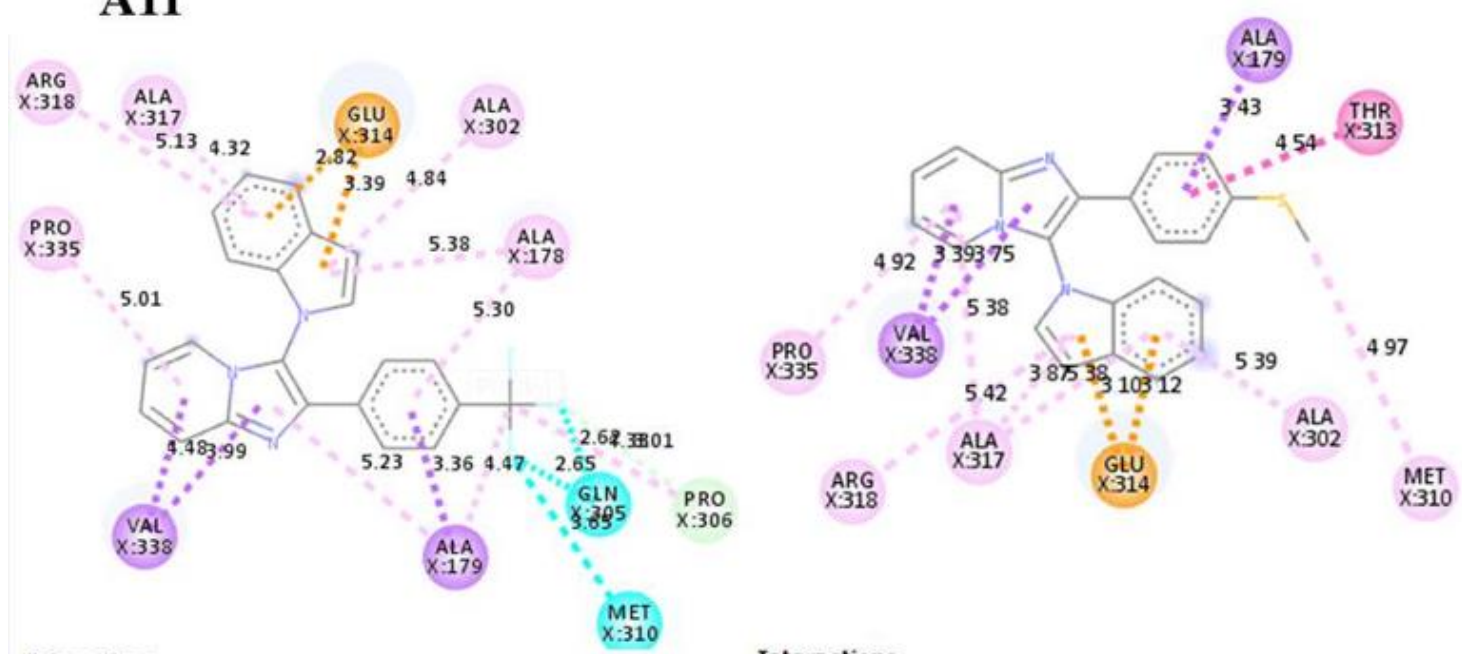

Interactions

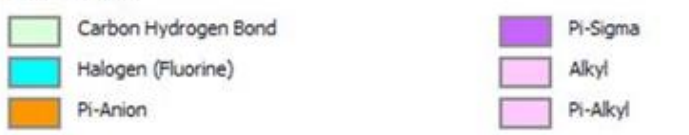

Interactions

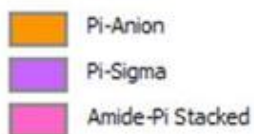

Alkyl

Pi-Alkyl 


\section{A9}

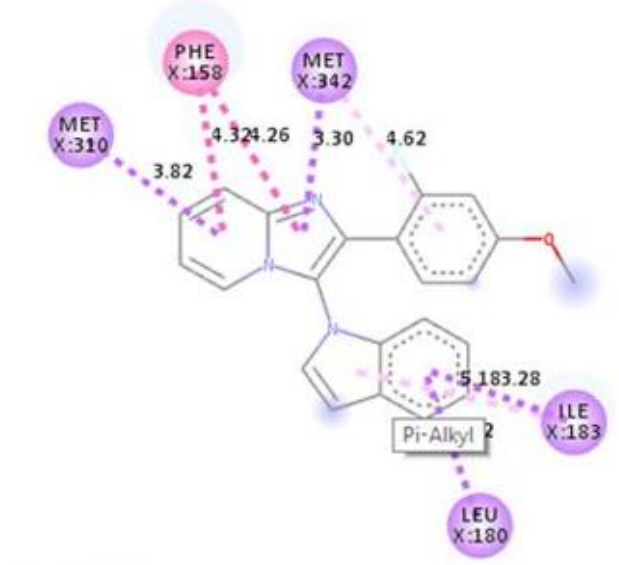

Interactions

Pi-Sigma

Pipi T-shaped

A13

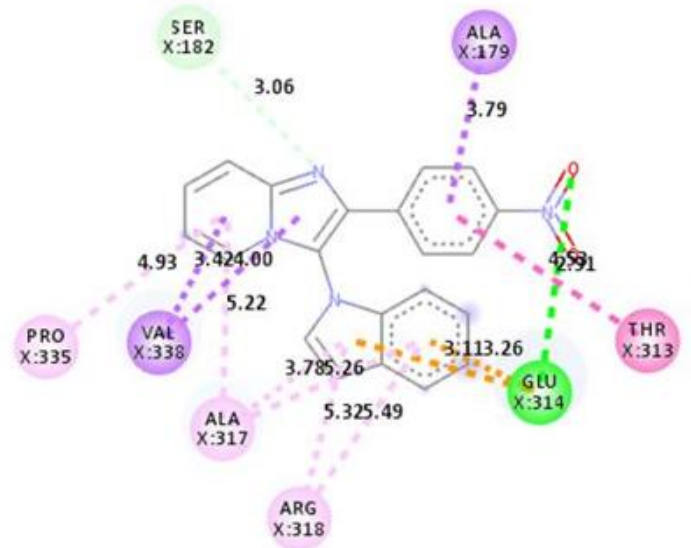

A10

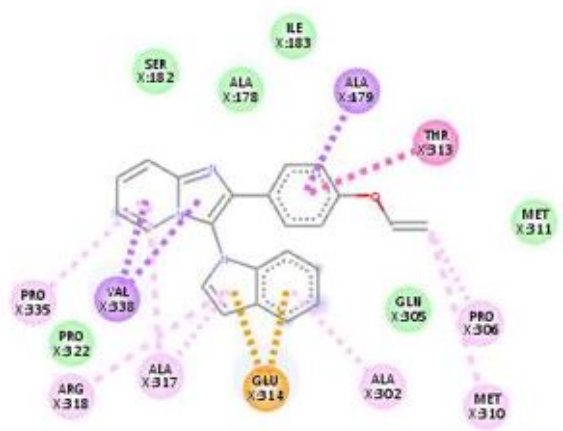

Interactions

$\square$ van der Waals
Pi-Anion
Pi-Sigma

A14

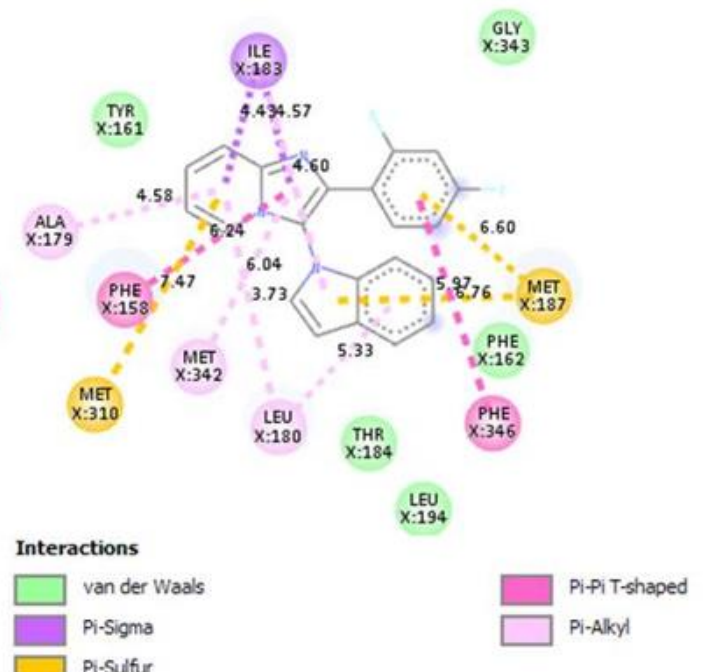

A15

$\underset{\mathrm{M}: 342}{\mathrm{ME}}$

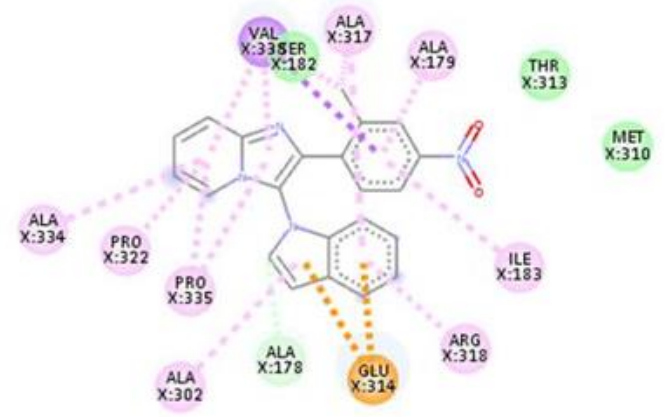

Interactions

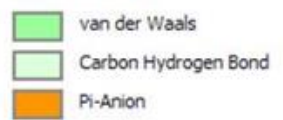

Pi-Sigma

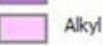

Pi.Alkyl

Figure S2. Molecular interaction illustrations of all compounds at active site of QcrB. 